\section{LA-UR-03-3/87}

Approved for public release; distribution is unlimited.

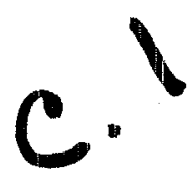

\section{Title: $\mid$ PHOTOINJECTOR RF CAVITY DESIGN FOR HIGH POWER CW FEL}

Author(s): Sergey S. Kurennoy, LANSCE-1

Dale L. Schrage, LANSCE-1

Richard L. Wood, LANSCE-1

Lloyd M. Young, LANSCE-1

Thomas Schultheiss, AES

Vincent Christina, AES

John Rathke, AES

Submitted to:

2003 Particle Accelerator Conference (PAC2003)

Portland, Oregon

May 12-16,2003

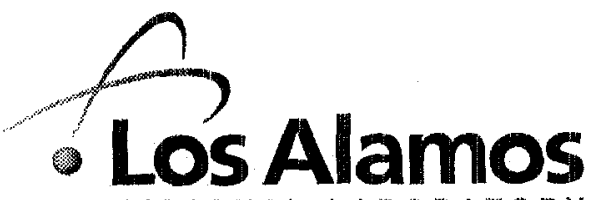

NATIONAL LABOAATORY

Los Alamos National Laboratory, an affirmative action/equal opportunity employer, is operated by the University of California for the U.S. Department of Energy under contract W-7405-ENG-36. By acceptance of this article, the publisher recognizes that the U.S. Government retains a nonexclusive, royalty-free license to publish or reproduce the published form of this contribution, or $\equiv$ ow others to do so, for U.S. Government purposes. Los Alamos National Laboratory requests that the publisher identify this article as wd $\square$ sformed under the auspices of the U.S. Department of Energy. Los Alamos National Laboratory strongly supports academic freedom and a researcher's right to publish; as an institution, however, the Laboratory does not endorse the viewpoint of a publication or guarantee its technical correctness. 


\title{
PHOTOINJECTOR RF CAVITY DESIGN FOR HIGH POWER CW FEL*
}

\author{
S.S. Kurennoy, D.L. Schrage, R.L. Wood, L.M. Young, LANL, Los Alamos, NM 87545, USA \\ T. Schultheiss, V, Christina, J. Rathke, Advanced Energy Systems, Medford, NY 11763, USA
}

\section{Abstract}

The project is under way to develop a key enabling technology for high-power CW FEL: an RF photoinjector capable of producing continuous average current greater than $100 \mathrm{~mA}$. The specific aim is a $\pi$-mode, normalconducting $\mathrm{RF}$ photoinjector, $3 \mathrm{nC}$ of bunch charge, 100 $\mathrm{mA}$ of current (at 33.3-MHz bunch repetition rate) and emittance less than $10 \mathrm{~mm}$-mrad. This level of performance will enable robust 100-kW-class FEL operation with electron beam energy $<100 \mathrm{MeV}$, thereby reducing the size and cost of the FEL. This design is scalable to the MW power level by increasing the electron bunch repetition rate to a higher value. The major challenges are emittance control and high heat flux within the CW $700-\mathrm{MHz}$ RF cavities. Results of RF cavity design and cooling schemes are presented, including both high-velocity water and liquid-nitrogen cooling options.

\section{INTRODUCTION}

For a MW-class FEL, a high-current emittance-compensated photoinjector is a key element. This paper presents results on the RF cavity design and cooling schemes for a demo 700-MHz RF photo-injector (PI) accelerating 100 $\mathrm{mA}$ of the electron beam ( $3 \mathrm{nC}$ per bunch at $33.3-\mathrm{MHz}$ repetition rate) to above $5 \mathrm{MeV}$ [1]. Further accelerating and focusing provides the transverse rms emittance below $10 \mathrm{~mm}$-mrad at the wiggler. The beam energy spread should be below $1 \%$ to allow the bunch compression down to 20 ps before the wiggler. The PI is designed to be scalable to higher beam currents, up to $1 \mathrm{~A}$, by increasing the bunch repetition rate. To keep the beam emittances low, high electric-field gradients, especially near the photocathode, are needed, plus an external magnetic field to compensate space charge. However, the wall power losses increase as cavity fields squared, for a given cavity shape, leading to serious challenges for cavity cooling and increasing the RF power required. Therefore, an optimal design must be a trade-off between the requirements of beam dynamics, RF power, and feasibility of the cavity thermal management.

\section{RF CAVITY}

We consider a $(n+1 / 2)$-cell, $\pi$-mode RF structure, with cell-to-cell coupling of 0.03-0.05. A few options for the cell coupling have been studied [2], including magnetic coupling via slots and more conventional on-axis electric coupling through apertures. It was found that using slots provides effective structures with high shunt impedance, but leads to very high power-loss densities near the slots. As the result, the on-axis coupled cavity structure was chosen [2]. This structure has an additional advantage: its septa (cell-separating walls) are easier to cool than those

* Supported by the DoD High Energy Laser Joint Technology Office through a contract from NAVSEA. with coupling slots. An emittance-compensating external solenoid, as well as a bucking coil that cancels its magnetic field near the cathode, have designs similar to those for the 1.3-GHz AFEL [3]. Superfish-Poisson codes [4] have been used for 2D and the CST MicroWave Studio (MWS) [5] for 3D electromagnetic calculations. Beam dynamics has been simulated with Parmela [6] to select field configurations minimizing the emittance for a given cavity shape. We found that a long structure, $n=6$ or 7 , is needed to achieve the beam energy of $5 \mathrm{MeV}$ while keeping the heat flux manageable. However, fields in such a long cavity would be unstable, with a rather small mode separation. Our solution was to split the structure. The resulting two-stage configuration consists of a 2.5-cell cavity with the accelerating gradient $E_{0}=7 \mathrm{MV} / \mathrm{m}$ (the PI proper) that brings the electron beam energy to $2.7 \mathrm{MeV}$, followed immediately by a conventional 4-cell booster with $E_{0}=4.5 \mathrm{MV} / \mathrm{m}$, where the beam is accelerated to 5.5 MeV. Results of Parmela simulations presented in Fig. 1 confirm that this design satisfies all beam requirements.

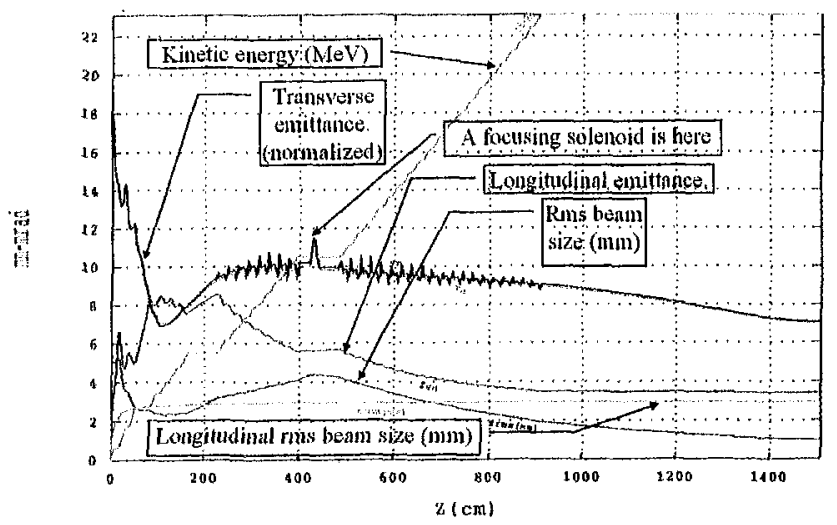

Figure 1: Results of Parmela simulations for the split PI.

The wall power density in the 4-cell booster, due to its lower gradient, is below $35 \mathrm{~W} / \mathrm{cm}^{2}$, so that its cooling will not present problems. The most challenging part of the PI is the 2.5-cell RF cavity. Its schematic, including cooling channels and RF tapered ridge-loaded waveguides, is shown in Fig. 2. With aperture radii of $65 \mathrm{~mm}$ and septum thickness of $20 \mathrm{~mm}$, the cell coupling in the 2.5 -cell structure is near 0.03 , so that the $\pi$-mode at $700 \mathrm{MHz}$ is well separated from its nearest neighbors. The cavity design is a result of a few iterations made with Superfish (SF) and the MWS eigensolver, followed by a thermal analysis. SF runs were used to adjust the cavity radii to achieve a flat field distribution, which gives minimal emittances at the wiggler. MWS eigenmode computations included 3D effects, like frequency shifts due to vacuum ports, RF couplers, or slug tuners. Time-domain simulations with MWS have been used to design and optimize the RF waveguide-cavity couplers [7]. 


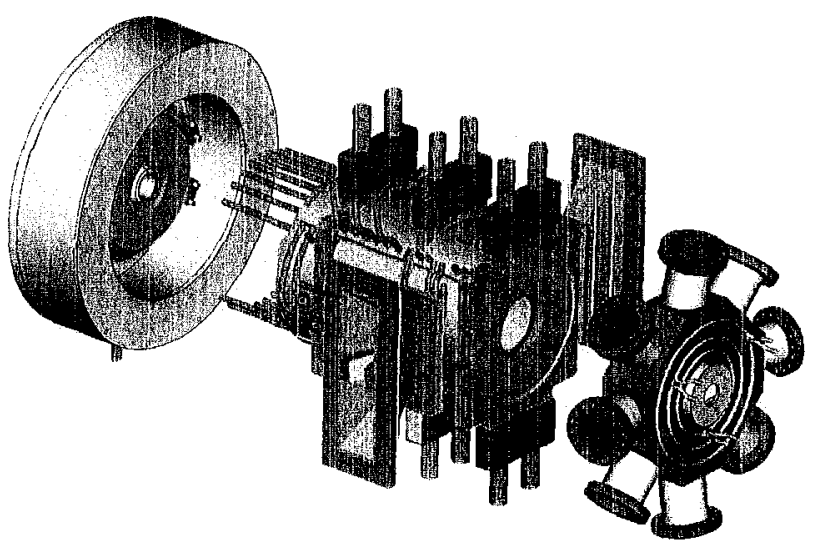

Figure 2: 2.5-cell RF cavity (center) with emittancecompensating magnets (left) and vacuum plenum (right).

The electric field of the $\pi$-mode in the PI 2.5-cell RF cavity is shown in Fig. 3 produced by MWS. The field magnitude corresponds to the MWS solver normalization of eigenmodes (the mode field energy is $1 \mathrm{~J}$ ), so it should be scaled (by a factor of 2.38) to provide the required gradient of $7 \mathrm{MV} / \mathrm{m}$. One can see how the field alternates its direction from one cell to the next one. The vacuum plenum (shown in front in Fig. 3) is not excited since its resonance frequency is much lower, at about $650 \mathrm{MHz}$. Its downstream wall will also house the laser window.

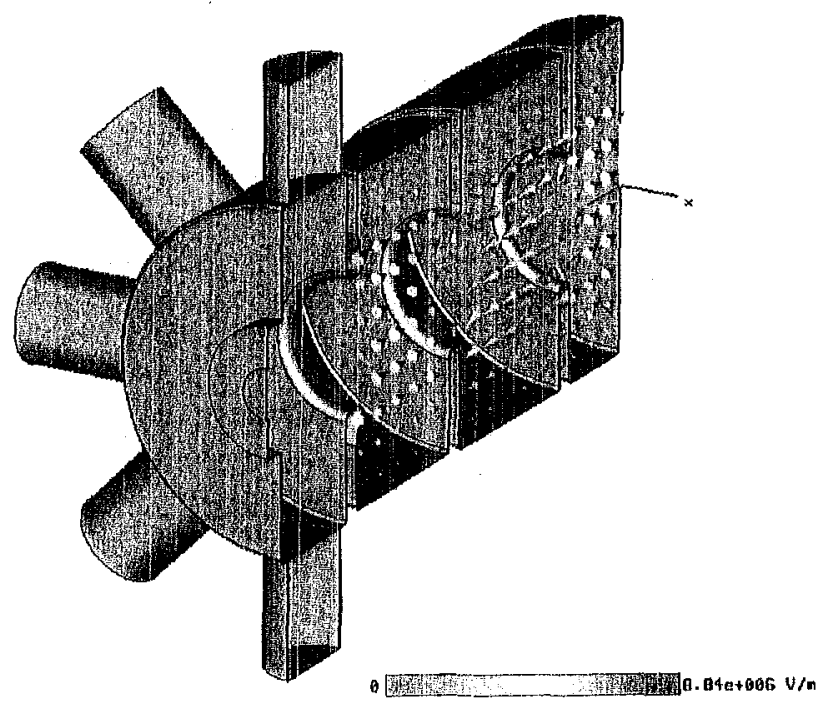

Figure 3: Electric field of $\pi$-mode in 2.5-cell PI RF cavity.

Wall power densities have been calculated using both codes, SF and MWS. The surface current distribution inside the cavity is illustrated in Fig. 4. The same field scaling as in Fig. 3 is assumed. The highest power density is on the cell septa and particularly on the $1^{\text {st }}$ half-cell end-wall, where it reaches slightly above $100 \mathrm{~W} / \mathrm{cm}^{2}$ for the nominal gradient $E_{0}=7 \mathrm{MV} / \mathrm{m}$. The power density increases even higher, above $200 \mathrm{~W} / \mathrm{cm}^{2}$, near the $\mathrm{RF}$ couplers connected to the $3^{\text {rd }}$ cell (cf. Fig. 2), see [7] for details. However, these hot spots are small and can be cooled efficiently using special cooling channels placed near the coupler iris in the thick cavity wall.

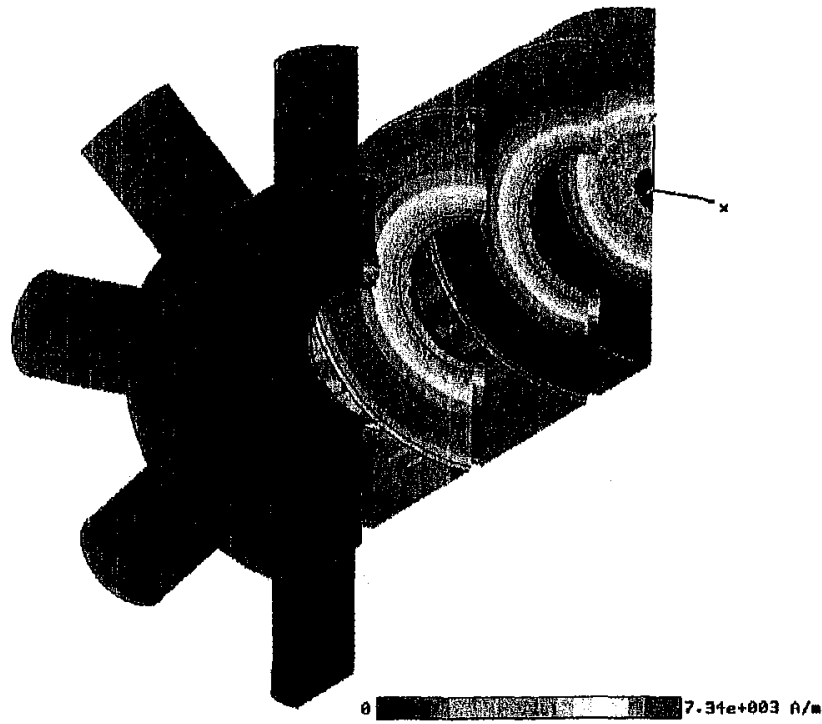

Figure 4: Surface current distribution for the $\pi$-mode.

\section{THERMAL MANAGEMENT}

The required high field operation of this cavity results in high RF losses on the surface. While cooling with liquid nitrogen $\left(\mathrm{LN}_{2}\right)$ as opposed to water would lower the wall loads significantly (Tab. 1), the cost of a highpressure liquid nitrogen system was prohibitive for the demonstration photoinjector. A high-pressure system ensures that boiling does not occur at these high heating rates. A forced flow / boiling cooling system, though cheaper, was considered too risky. Therefore, a watercooled design was pursued. This design, with some tuning to adjust the frequency (e.g., with slug tuners in the $2^{\text {nd }}$ cell of the 2.5-cell cavity), would also work with highpressure liquid nitrogen if desired.

Table 1: RF losses at $\mathrm{LN}_{2}$ and room temperature

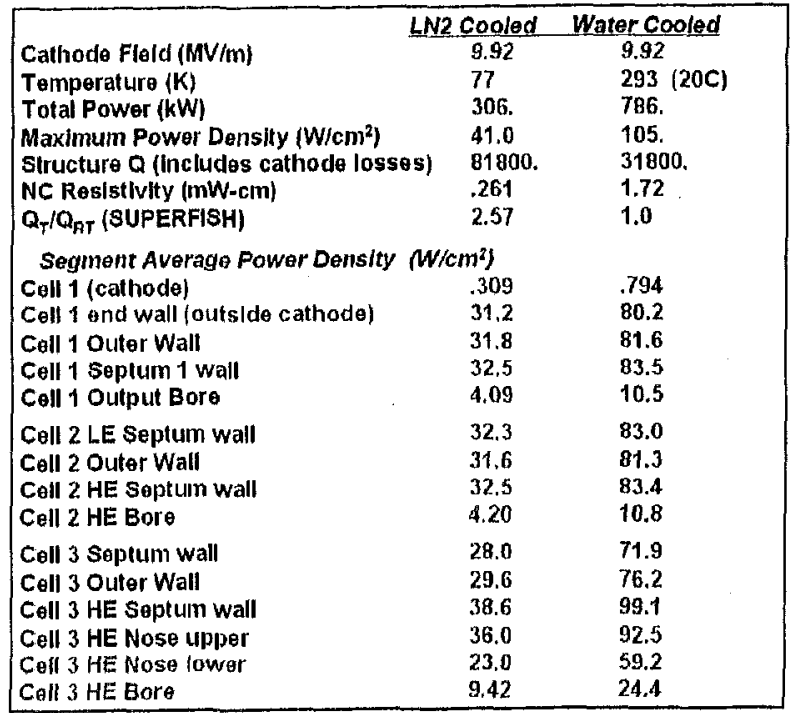

About eight hundred kilowatts of RF power are deposited on the walls of the 2.5-cell cavity. This requires intricate cooling channels and a relatively large flow of cooling water. Cooling tubes for the cylindrical walls and 
cooling plenums for the septa can be seen in Fig. 2. Flow requirements that are based on RF heat loads at surface temperature are given in Tab. 2 .

Table 2: Flow requirements for 2.5-cell RF cavity

\begin{tabular}{|l|l|}
\hline Total RF ohmic loss* & $823 \mathrm{~kW}$ \\
\hline Max surface heat flux* & $112 \mathrm{~W} / \mathrm{cm}^{2}$ \\
\hline Water inlet temperature & $20 \mathrm{C}$ \\
\hline Coolant channel dimensions & $.100^{\prime \prime} \times .175^{\prime \prime}(2.5 \times 4.5 \mathrm{~mm})$ \\
\hline Flow rate & $36 \mathrm{l} / \mathrm{s}$ at $5 \mathrm{~m} / \mathrm{s}$ \\
\hline RF surface temperature & $52 \mathrm{C}$ \\
\hline Coolant channel surface temp. & $46 \mathrm{C}$ \\
\hline Pressure drop & $15.8 \mathrm{psi}$ within channel, \\
\hline Mean coolant outlet temp. & $50 \mathrm{psi}$ overall \\
\hline
\end{tabular}

* At RF surface temp, OFE Cu surface

An axisymmetric model of the cavity was developed using the finite element code ANSYS. RF loads were mapped on the surface of the model from Superfish output and modified based on the surface temperature. The resulting steady-state temperature distribution is shown in Fig. 5. The left cell is the half cell with the photocathode on the far left wall. The septa separating the cavity cells have simple shapes to allow for simple coolant channel design. The fourth cell (far right) is the non-resonant vacuum pumping cell. The RF losses are relatively small on this cell, so only limited cooling is required.

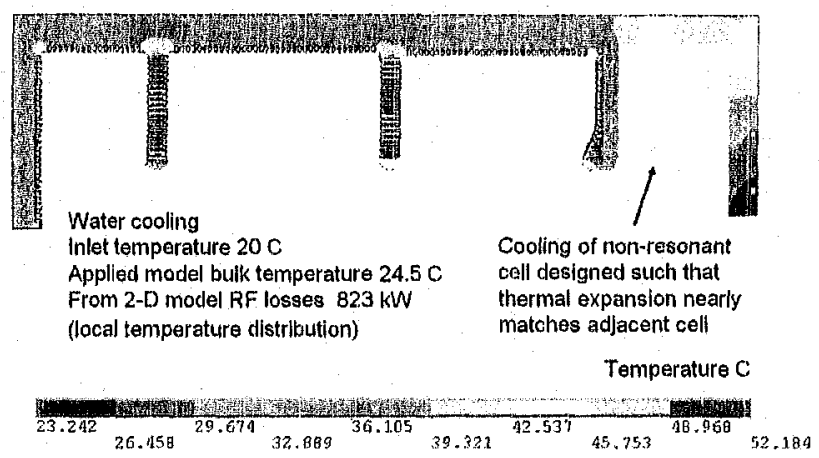

Figure 5: Steady-state temperature distribution.

The significant stresses generally occur between the heated RF surface and the cooling channels. GlidCop( $(1)$, a dispersion strengthened copper, is used in the high stress regions throughout this cavity. It has a yield strength of 39 $\mathrm{ksi}$ and provides significant margin for this design. The steady-state temperature distribution is subsequently mapped onto a structural finite element model using the same nodes and element connectivity as the thermal model. The resulting steady-state von Mises stress distribution is shown in Fig. 6.

The PI is designed to be a fully hydrogen furnace brazed structure. The core of the structure will be fabricated from Glidcop $\otimes$ AL-15 with some external elements made from OFE copper and stainless steel. The double-ridged waveguide tapers are also made from Glidcop ${ }^{\circledR}$. The majority of the complex machining required for the cooling channels will be done in the Glidcop $^{\circledR}$ structures. They will then be copper plated to facilitate assembly by brazing. Final cuts on the inside diameter of the cells will be done to establish the desired field profile and frequency prior to stack brazing the cavity assembly.

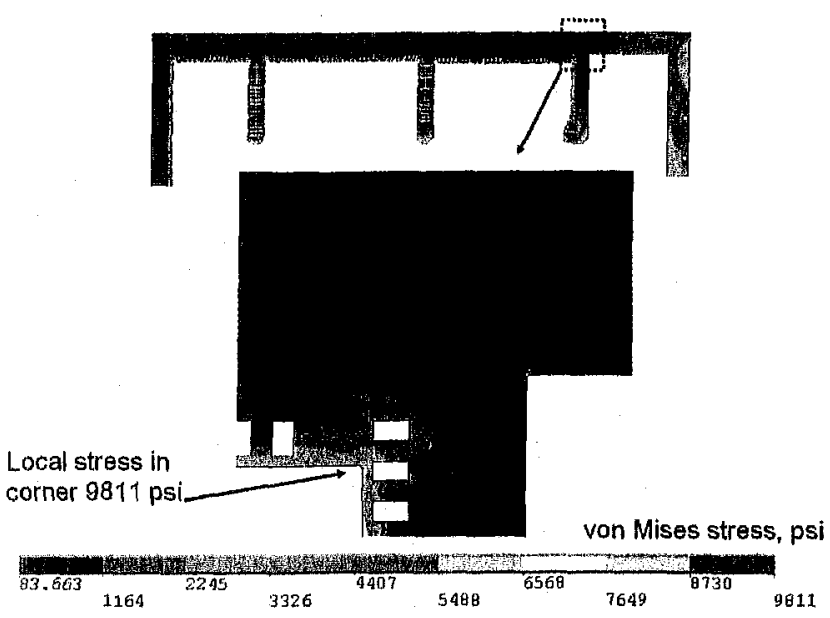

Figure 6: Steady-state thermal stress distribution.

\section{CONCLUSIONS}

The presented design allows us to surpass the required beam parameters while addressing the key issue for a high-current normal conducting CW RF photoinjector, namely, an effective structure cooling. It provides a path forward to a very high power amplifier FEL.

We plan to construct the full-power prototype, install it in the existing facilities at LANL, and to perform RF and thermal testing without beam in early 2005. Once the $\mathrm{RF} /$ thermal management with high heat flux has been demonstrated, the system will be upgraded to operate with electron beam, by adding a real photocathode and drive laser, a second RF power source, and a short beam line with a beam dump and appropriate diagnostics.

\section{REFERENCES}

[1] D.C. Nguyen, et al. "Development of a CW, HighAverage-Current $700 \mathrm{MHz}$ Photoinjector at Los Alamos", FEL'02, Argonne, IL, Sep. 2002.

[2] S.S. Kurennoy, et al. "CW RF Cavity Design for High-Average-Current Photoinjector for High Power FEL", ibid.

[3] R.L. Sheffield, et al. NIM, A318 (1992) 282.

[4] J.H. Billen, L.M. Young. Report LA-UR-96-1834, Los Alamos, 1996 (rev. 2002).

[5] MicroWave Studio, v.4.2. CST GmbH, www.cst.de.

[6] L.M. Young, J.H. Billen. Report LA-UR-96-1835, Los Alamos, 1996 (rev. 2002).

[7] S.S. Kurennoy, L.M. Young. "RF Coupler for HighPower CW FEL Photoinjector", these proceedings. 